\title{
Review
}

\section{Research Progress on Effects of Regulatory B Cells in Infection Immunity}

Ting Miao

Department of Pharmacy, Tianjin $4^{\text {th }}$ Center Hospital, Tianjin China

\section{Keywords \\ Regulatory B cells; infection immunity; IL-10; TGF- $\beta$}

\section{Correspondence}

Ting Miao,

E-mail: tingmia082@163.com

DOI: 10.1515/ii-2017-0124

\begin{abstract}
B cells play immunomodulatory roles mainly by presenting antigens and producing antibodies. In recent years, some B cells were shown to exhibit regulatory functions. This type of B cell was named regulatory B cells (Bregs). Bregs can mediate immune tolerance to inhibit excessive inflammatory responses and to accelerate recovery of inflammation by producing interleukin 10 and/or transforming growth factor $\beta 1$ and other inhibitory cytokines. Studies showed that Bregs play important roles in parasites, bacteria, and viral infections. This study reviews biological characteristics, functions, and microsignal regulation of Bregs and their mechanism in infectious diseases and related research progress.
\end{abstract}

B cells play a central role in humoral immunity by regulating CD4+ $\mathrm{T}$ cell responses. These cells can also be used as antigen-presenting cells (APCs) and produce cytokines, including interleukin (IL)-4, IL-6, IL-10, and transforming growth factor- $\beta$ (TGF- $\beta$ ) to provide costimulatory signals that promote differentiation of CD4+ T cells into helper T cell (Th1 or Th2) subgroups ${ }^{[1]}$. However, B cells perform additional immune functions, including production of cytokines, which act as secondary APCs. Regulatory B cells (Bregs) feature protective effects that inhibit immune responses ${ }^{[2]}$. Subgroups of Bregs mainly include the CD1d ${ }_{\text {hi }}$ CD5 + CD19 ${ }_{\text {hi }}$ phenotype. B10 cells represent subgroups of Bregs with CD1d ${ }_{h i}$ CD5+ CD19 ${ }_{\text {hi }}$ phenotype and produce IL-10. Mizoguchi et al. ${ }^{[2,3]}$ first proposed Bregs and described that these B cell subgroups exhibit regulatory functions. Janway et al. observed that lack of Bregs in B10/PL mice caused development of severe experimental autoimmune encephalomyelitis (EAE), suggesting that Bregs are produced and developed in EAE mouse model by producing IL-10 that affects occurrence and development of autoimmune diseases ${ }^{[3-5]}$.

\section{Definition of Bregs}

Bregs are derived from B cells, and they can inhibit acquired immune-mediated inflammatory responses and/or accelerate recovery of inflammation by directly producing IL-10 and TGF- $\beta 1$ with secondary antigen presentation or through secretion of antibodies that interact with other immune cells $[2,6]$.

\section{Source, classification, and function of $B$ cells}

Hongbo et al. ${ }^{[6,7]}$ reported that Bregs can be divided into primitive type and acquired type. Primitive Bregs mainly originate from mesenteric lymph nodes. Intestinal bacteria produce a large number of metabolites and can induce primitive Bregs to differentiate into acquired Bregs, thus inducing a series of immunosuppressive effects. B cell development can be divided into preB cells, immature B cells, mature B cells, activated B cells, and plasma cells. Differentiation of preB cells and immature B cells is antigenindependent and occurs in bone marrows. Differentiation of immature B cells, activated B cells, and plasma cells is antigen-dependent and occurs in peripheral organs ${ }^{[8,9]}$. According to different surface molecules, B cells are divided into B1 and B2 cells. B2 cells are commonly referred to as $B$ cells. B1 cells reside in the chest and abdominal cavity and are formed in the fetal liver. Peritoneal B1 cells express Mac-1 (CD11b), whereas spleen B1 does not. Regardless 
of absence or presence of CD5 expression, B1 cells can further differentiate into B1a cells (CD11b+ CD5+) and B1b cells (CD11b+CD5-). B2 cells mainly exist in secondary lymphoid organs and can continually multiply in adult bone marrows ${ }^{[10]}$.

Mizoguchi et al. ${ }^{[2,11]}$ observed that Bregs act as secondary APCs by producing IL-10, which inhibits immune responses. IL-10 can also regulate the balance of TH1 and $\mathrm{TH} 2$ cells. Bregs activate CD4+ T cells and natural killer cells to induce immunosuppressive effects.

\section{Microenvironment signals and production of Bregs}

To date, common surface markers of Bregs or specific transcription factors, both in humans and in mice, remain unidentified. Appropriate stimulus can produce Bregs; this concept can be reinforced as follows: some factors that exist in microenvironment may play important roles in induction of B cells ${ }^{[12]}$. Toll-like receptor (TLR) agonists effectively inhibit B cell induction. Lipopolysaccharides (LPS) in Gramnegative bacteria can induce Bregs to produce IL-10, thereby inhibiting disease progression in EAE mouse models ${ }^{[13]}$. Mice cannot recover from EAE because of lack of primary reaction gene 88 with differentiation of B cells, TLR2, TLR4 or TLR myeloid cells; this phenomenon indicates that TLR is directly involved in regulatory $B$ cell regulation function [3,14]. TLR signal enables naive B cells to produce IL-10, whereas other B cells still need ligation reaction of CD40 and $B$ cell receptor (BCR) to further produce IL-10 ${ }^{[15]}$. During the initial stage, TLR only induces a small amount of Bregs. The second stage further stimulates production of Bregs as a result of ligation reaction of BCR and CD40, eventually leading to production of sufficient amounts of IL-10 ${ }^{[13]}$. Bouaziz et al. ${ }^{[16]}$ also confirmed that TLR9 strongly stimulates IL-10 production. These results indicate that TLRs play important roles in induction of Bregs. Gray et al. ${ }^{[17]}$ suggested that apoptotic cells can also trigger IL-10 production as an endogenous signal to improve collageninduced arthritis. Apoptosis can also induce spleen cells to produce IL-10. Enhanced T cell secretion of IL-10 may play an immunosuppressive function ${ }^{[3]}$.

\section{Function of Bregs in parasitic infection}

Harris et al. ${ }^{[18]}$ showed that B cells, such as CD4+ T cells, can produce cytokines under conditions of inflammation caused by Toxoplasma gondii or polymorphic helical nematode infection. In schistosomiasis-infected mice, Bregs may be associated with changes in TH1 and TH2 and are related to $\mathrm{CD} 4+\mathrm{T}$ cells that depend on pathology of granulomatosis caused by parasite eggs. Jankovic et al. ${ }^{[19,20]}$ studied regulation mechanism of B cells in schistosomiasis infection. Although development of parasites in wild-type (WT) mice and B cell-deficient hosts were similar, survival rate of B-cell deficient mice significantly decreased. In this study, pathological phenomena of hepatic granulomatosis increased, showing similarity to conditions of mice with B cell deficiency and Fc receptor deficiency. This finding suggests that granulomas formed by the body's resistance against parasitic diseases are associated with antibodies produced by B cells. However, sensitivity test for detecting schistosomiasis infection in B cell-deficient mice does not include Fc receptor-deficient animals. This phenomenon suggests that antibodies are only partially involved in B cell-mediated regulatory process, and Bregs may also be involved. Other studies showed that Bregs producing IL-10 can partially limit pathological development of granuloma. Components of lacto-N-fucose pentoxide III and Schistosoma mansoni soluble egg antigens (SEA) stimulate B cells ${ }^{[21]}$, especially B1 cells [22], to produce IL-10. However, in the first five weeks of abdominal infection, schistosomiasis infection is associated with overexpression of CD5+ B1 cells [22]. This effect is similar to that of lacto- $\mathrm{N}$-fucose pentoxide III-coated magnetic beads, which shows that the important role of eggs in the body is related to carbohydrate of $B$ cells. However, further studies should determine whether BCRs, triggering of specific lectins, or both are involved in activation of B cells. The role of SEA with several exogenous lectins can regulate natural responses of dendritic cells (DC) ${ }^{[23]}$. The role of B1 cells in parasite infection was manifested in a mouse model with CD5+ B1 cell deficiency and a model of Bruton's tyrosine kinase deficient X-linked immunodeficiency (XID) mice ${ }^{[24]}$. Thus, XID mice exhibit high sensitivity to parasites, such as Schistosoma mansoni ${ }^{[25]}$. For parasitic diseases, phosphorylcholine on L3 antigens increases the number of $\mathrm{B} 1$ cells that produce IL-10 and also reduces intensity of antibody responses ${ }^{[26]}$. Thus, in both cases, susceptibility is associated with decreases in IL-10 and antibody responses. Recently, Laleh et al. ${ }^{[20,27]}$ established an experimental model using schistosomiasis-infected Bregs to 
treat mice allergies. In this previous study, B cell transfection from WT-infected mice, but not IL-10-deficient mice, showed protection against the effects of allergic reactions, further demonstrating immunomodulatory effects of Bregs.

\section{Role of Bregs in bacterial and viral infections}

LPS on cell wall of Gram-negative bacteria can display antiinflammatory properties of B cells. Thus, possibilities of regulatory role of Bregs in bacterial infection are increased. Recent studies showed that amounts IL-10 produced by CD5+ B (relative to LPS and agonist reactions of TLR2, 5, 7,8 , and 9) agree with high TLR expression levels in B cells ${ }^{[28,29]}$. This finding also confirms literature conclusion ${ }^{[30]}$, which implies that $\mathrm{B}$ cells in the spleen margin region produce IL-10 after TLR activation, and these cells can also produce large amounts of IL- $6^{[20]}$.

Cytokines in B cells possibly aid specific B cell functions, such as cell survival and cell differentiation. Under these conditions, one possible role of CD5+ B-cell-derived IL-10 in TLR activation is to limit proinflammatory response of TLRinduced myeloid cells. This hypothesis supports a recent study data, which indicated that IL-10 produced by CD5+ $B$ cells can downregulate level of IL-12 ${ }^{[20,29]}$ produced by TNF- $\alpha$, IL-6, and DC. In vivo, new neonatal mice presented regulatory mechanisms of Bregs in systemic inflammatory responses. B1 cells were produced in liver-derived precursors and matured during the fetal period ${ }^{[31]}$. During the neonatal period, the liver still largely contributes to hematopoietic effect of B cells, leading to expansion of B1 cells to the periphery, whereas bone marrow-derived B2 cells initiate production. Therefore, in the context of TLR activation, regulatory $\mathrm{CD} 5+\mathrm{B}$ cells can strongly inhibit neonatal innate inflammation. The early role of neonatal CD5+ B cells is release of IL-10 followed by activation of TLR ligands; this phenomenon results from early control of DC TH1 cells, leading to decreased IL-12 and neonatal Th2 type immune bias ${ }^{[28]}$. These results emphasize effects of various types of Bregs and TLR triggering observed in vivo.

Bregs can limit TLR7 and TLR9 ligand effects in newborns ${ }^{[29]}$. Type I interferon (IFN) plays an important role in TLR-activated bone marrow cells. However, the initial type I IFN plays a key role in IL-10, which is produced by neonatal Bregs. Migration of IL-10 can protect CD5+ $\mathrm{B}$ cells in $\mathrm{IL}-10^{-/-}$neonatal mice from destruction of inflammatory responses caused by $\mathrm{CpG}$ (tandem repeats sequences consisting of cytosine $\mathrm{C}$ and guanine $\mathrm{G})^{[29]}$. By contrast, this condition exhibits no effect on protecting IL- $10^{-/-}$neonatal mice with IFN $\alpha / \beta$ receptor deficient CD5+ $B$ cells. IL-10 produced by synergistic effects of I-type IFN and natural CD5 + B cells plays a key role in this process and helps neonatal mice in preventing excessive inflammatory responses. Bregs affect inflammation caused by I-type IFN and preTH1 in neonatal mice. This phenomenon suggests that Bregs may contribute to increasing susceptibility to pathogens during the early period of infection. Responses to TLR9 and TLR7 triggered by influenza virus and baculoviruses, such as granuliform virus and others, support the ability of CD5+ B cells to produce IL-10. Response of IL-10 to viruses further increases ability of plasma cell DC reactions to produce IFN- $\alpha$ compared with that of the same viruses ${ }^{[20,29]}$. However, in the fight against viruses and bacterial infections, positive effects of TLR activation of Bregs should be recognized ${ }^{[32,33]}$. Regulation and protection of Bregs should be studied in vitro and in bacterial infections.

\section{Outlook}

Bregs exhibit significant antiinflammatory effects during parasitic infection. IL-10, which is secreted by Bregs, transfers immune responses from Th1 to Th2. Although studies on Bregs achieved periodic progress, many problems still exist and must be solved. For example, further studies should center on cytokine secretion of Bregs by signal transduction, whether Bregs feature independent regulatory cellular mechanisms such as those of Tregs, in vivo Bregs phenotypes, and their transcription factors.

\section{Declarations}

\section{Acknowledgements}

No.

\section{Competing interests}

The author declares that she has no competing interest.

\section{Authors' contributions}

T Miao made the literature analysis and wrote, discussed and revised the manuscript of this review.

\section{References}


1 Koichi Yanaba, Jean-David Bouaziz, et al. A regulatory B cell subset with a unique CD1dhiCD5+ phenotype controls $\mathrm{T}$ cell-dependent inflammatory responses. Immun, 2008, 28(5):639-50.

2 Mizoguchi A, Bhan AK. A case for regulatory B cells. J Immun, 2006,176(2):705-10.

3 Yang M, Rui K, Wang SG, et al. Regulatory B cells in autoimmune diseases. Cell Mol Immun, 2013, 10(13):122-32

4 Wolf SD, Dittel BN, Hardardottir F, et al. Experimental autoimmune encephalomyelitis induction in genetically B cell-deficient mice. J Exp Med, 1996, 184(6):2271-8.

5 Fillatreau S, Sweenie CH, McGeachy MJ, et al. B cells regulate autoimmunity by provision of IL-10. Nat Immun, 2002, 3(10):944-50.

6 Liu H, Feng Y, Zheng X. Study on Regulatory B Cells. Journal of Tropical Medicine, 2012, 12(5): 636-8.

7 Mizoguchi A, Mizoguchi E, Smith RN, et al. Suppressive role of B cells in chronic colitis of $\mathrm{T}$ cell receptor mutant mice. J Exp Med, 1997, 186(10):1749-56.

8 Depraetere S, Verhoye L, Leclercq G, et al. Human B cell growth and differentiation in the spleen of immunodeficient mice. J Immun, 2001,166(5):2929-36.

9 Hu X, Yang W, Tian F. Regulatory B Cells and Methods Immunoregulation of Helminths. Chinese Journal of Pathogen Biology, 2013, 8 (3): 281-4.

10 Littman DR, Rudensky AY. Thl7and regulatory T cells in medjating and restraining inflammation. Cell, 2010, 140(6):845-58.

11 Fillatreau S, Gray D, Anderton SM. Not always the bad guys: B cells as regulators of autoimmune pathology.Nat Rev Immun, 2008, 8(5):391-7.

12 Gray D, Gray M. What are regulatory B cells. Eur J Immunol, 2010, 40(10):2677-9.

13 Lampropoulou V, Hoehlig K, Roch T, et al. TLR-activated B cells suppress T cell-mediated autoimmunity.J Immun, 2008, 180(7):4763-73.

14 Evans JG, Chavez-Rueda KA, Eddaoudi A, et al. Novel suppressive function of transitional 2Bcells in experimental arthritis. J Immun, 2007, 178(2):7868-78.

15 Cross AH, Trotter JL, Lyons J. B cells and antibodies in CNS demyelinating disease .J Neuroimmunol, 2001, 112(1-2):1-14.

16 Bouaziz JD, Calbo S, Maho-Vaillant M, et al. IL-10produced by activated human B cells regulates CD4+ T-cell activation in vitro. Eur J Immun, 2010, 40(10):2686-91.

17 Gray M, Miles K, Salter D, et al. Apoptotic cells protect mice from autoimmune inflammation by the induction of regulatory B cells .Proc Natl Acad Sci USA, 2007, 104(35):14080-5.

18 Harris DP, Haynes L, Sayles PC, et al. Reciprocal regulation of polarized cytokine production by effector B and T cells. Nat Immunol, 2000, $1(6): 475-82$.

19 Jankovic D, Cheever AW, Kullberg MC, et al. CD4+T cellmediated granulomatous pathology in schistosomiasis is downregulated by a $\mathrm{B}$ cell-dependent mechanism requiring Fc receptor signaling. J Exp Med, 1998, 187(4):619-29.

20 Laleh M, Richard LM, Claude L. Regulatory B and T cells in infections. Mic Infec, 2008, 10(9):1030-5.

21 Velupillai P, Harn DA. Oligosaccharide-specific induction of interleukin 10 production by $\mathrm{B} 220+$ cells from schistosome-infected mice: a mechanism for regulation of CD4+T-cell subsets. Proc Nat Acad Sci U S A, 1994, 91(1):18-22.

22 Velupillai P, Secor WE, Horauf AM, et al. B-1cell(CD5+B220+) outgrowth in murine schistosomiasis is genetically restricted and is largely due to activation by polylactosamine sugars. J Immun, 1997, $6(1): 338-44$.

23 Liempt E, Vliet SJ, Engering A, et al. Schistosoma mansoni soluble egg antigens are internalized by human dendritic cells through multiple C-type lectins and suppress TLR-induced dendritic cell activation. Mol Immun, 2007, 44(10):2605-15.

24 Khan WN, Gerstein RM, Malynn BA, et al. Defective B cell development and function in Btk-deficient mice. Immun, 1995, 12(9):283-99.

25 Gaubert S, Maurage CA. X-linked immunodefi-ciency affects the outcome of Schistosoma mansoni infection in the murine model. Par Immun, 1999, 32(8):89-101.

26 Al-Qaoud KM, Fleischer B, Hoerauf A. The Xid defect imparts susceptibility to experimental murine filariosis-association with a lack of antibody and IL-10production by B cells in response to phosphorylcholine. Inter Immun, 1998, 10(1):17-25.

27 Mangan NE, Fallon RE, Smith P, et al. Helminth infection protects mice from anaphylaxis via IL-10-producing B cells. J Immun, 2004, 173(10):6346-56.

28 Sun CM, Deriaud E, Leclerc C, et al. Upon TLR9signaling, CD5+B cells control the IL-12-dependent Th1-priming capacity of neonatal DCs. Immun, 2005, 22(4):467-77.

29 Zhang X, Deriaud E, Jiao X, et al. Type I interferons protect neonates from acute inflammation through interleukin 10-producing B cells. J Exp Med, 2007, 121(4):1107-18

30 Walker WE, Goldstein DR. Neonatal B cells suppress innate toll-like receptor immune responses and modulate alloimmunity. J Immun, 2007, 179(3):1700-10

31 Hardy RR, Hayakawa K. B cell development pathways. Ann Rev Immun, 2001, 19:595-621

32 Baumgarth N, Herman OC, Jager GC, et al. B-1and B-2cellderived immunoglobulin $\mathrm{M}$ antibodies are nonredundant components of the protective response to influenza virus infection .J Exp Med, 2000, 192(2):271-80.

33 Boes M, Prodeus AP, Schmidt T, et al. A critical role of natural immunoglobulin $\mathrm{M}$ in immediate defense against systemic bacterial infection. J Exp Med, 1998, 188(12):2381-6. 